RAD Conference Proceedings, vol. 2, pp. 217-221, 2017

www.rad-proceedings.org

\title{
RECONSTRUCTION OF MaXEnt IMAGES FROM PET CAMERA
}

\author{
D. Üstünda $\breve{g}^{*}$
}

The University of Marmara, the Department of Mathematics, Istanbul, Turkey

\begin{abstract}
We study here one of the imaging techniques, used in nuclear medicine, called a Positron Emission Tomographic (PET) imaging that provides information about many biological processes that are essential to the functioning of the organ that is being visualized. Our emphasis is given to applications of the maximum entropy image reconstruction method called "Cambridge MaxEnt Package" (CMEP) for recovering images of the human brain from data obtained by PET camera. Computer simulations demonstrate its usefulness.
\end{abstract}

Key words: Image reconstruction, maximum entropy, inverse problems, ill-posed problems, back projection, regularization, regression, nuclear medical imaging

DOI: $10.21175 /$ RadProc.2017.44

\section{INTRODUCTION}

In the last few decades of the century, there have been significant advances in nuclear medicine. In particularly, when Allen Cormack [1] and Godfrey Hounsfield [2] introduced X-ray computer tomography (CT) independently in the early 1970 s based on the mathematical foundation by Radon [3] for reconstructing images of an object from its projections, the field of nuclear medicine was revolutionized. The breakthrough development of X-ray CT was made possible by continuing advances in instrumentation and computer technologies, which also accelerated the development of the multi-dimensional imaging modalities that carry a great potential for providing, in addition to morphologic, dynamic and functional information on biochemical and pathophysiological processes or organs of the human body. Recent advances in basic molecular and cell biology have also made us change in our understanding of diseases which can be defined as alternations in cellular behaviour that reflect functional changes instead of defining it as structural changes. Actually, it is evident that pathological conditions are easily visible in either a conventional CT or the latest scanners; the underlying biochemical abnormality is usually well advanced. This is because X-ray CT imaging is mainly concerned with anatomical structures of body, distinguishing between different components that have different absorbing power of X-rays. Nevertheless, the latest scanners can provide radically improved qualitative diagnostic information relevant to a wide range of human diseases but give limited information about the functional or physiological state of the internal organs of the human body. In medicine, it is known that human diseases often occur with no specific anatomical changes and those seen may be later effects of early biochemical process, which remain undetected until advanced symptoms appear in the patient. Therefore, the goal of the radiology specialty of nuclear medicine is to provide information on the distribution of a chosen molecule in space inside the human body, so that an image of its distribution within the body or a specific organ provides information on the functioning of the body or organ that is valuable for medical diagnosis. For this purpose, PET imaging techniques $[4,5,6,7]$ for measuring concentrations of positron-emitting radioisotope within the tissue of living subjects have been developed by researchers from diverse disciplines for the last decade. Since the first introduction of PET to medical imaging applications in the late 1960's, it has already grown into a well-researched, highly evolved field $[8,9,10]$.

Most of the current approaches to tackle the problem of the reconstruction of isotope concentration distribution can be classified into two general categories, namely the analytical methods [11, 12], which rely on the inversion of Radon transform, and the iterative approaches $[13,14,15,16]$, which are mainly based on a statistical description of the physical problem. Because of the random nature of radioactive disintegration, the tomographic data are noisy and therefore it is logical to regard PET reconstruction as a statistical estimation problem. Such approaches, when reconstructing PET images, require us to introduce modelling of the data statistics and to include a-priori constraints such as no negativity and smoothness. In this paper, we therefore focus on the image reconstruction method that is based on the Bayesian approach [17], because it permits the use of prior knowledge concerning the situation under study so that the incorporation of PET system model and

\footnotetext{
*dustundag@marmara.edu.tr
} 
measurement statistical uncertainties provide more robust and accurate PET images.

\section{Pet MEASUREMENT MODELLING}

In PET measurement, initially, when a positronemitting nuclide decays in a body, the nucleus rids itself of excess positive charge by emitting a positron, which almost immediately loses its energy by colliding with surrounding tissues, then it combines with an electron, and annihilates itself. Two back-to-back gamma rays with equal energy are then generated. Two opposite detectors can coincidently detect these photon pairs, $C_{1}$ and $\mathrm{C}_{2}$, externally, forming an annihilation coincidence event detection shown in Figure 1 . However, in reality, the coincidence events may also include those when two gamma rays originating from two unrelated positron annihilations are detected within the coincidence timing window $t$ and those when the annihilation photons lose their direction and energy before they arrive to the detector system.

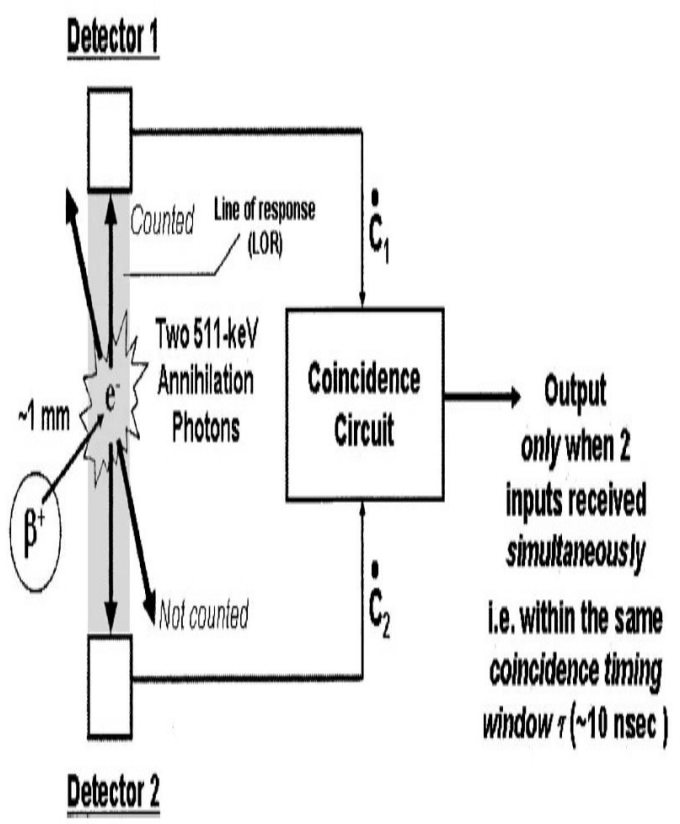

Figure 1. Basic principles of annihilation coincidence detection

Actually, the raw data recorded by a PET camera consist of $10^{6}$ random coincidence events, which are represented by a pair of coordinate values - one from each detector. Thus, image reconstruction may then proceed in two stages:

The first stage is to back-project the raw data. Then the second stage is to deconvolve the back-projected data with the point-spread function, shown in Figure 2 (b). It is a "spread" out version of the point source placed in the PET system and looks like a Gaussian shaped function with very long tails. To form a backprojection, the image volume (typically a cube $\left(600 \times 300 \times 124 \mathrm{~mm}^{3}\right)$ is divided into $K$ equally spaced planes, each of which consists of $\left(256^{\prime} 128\right)$ squared elements (pixels). Then, simple back-projected data is obtained by a computer program which forms a line of response between a pair of coordinate values recorded as raw data and increments the count in the pixel where this line passes in each back-projection plane.

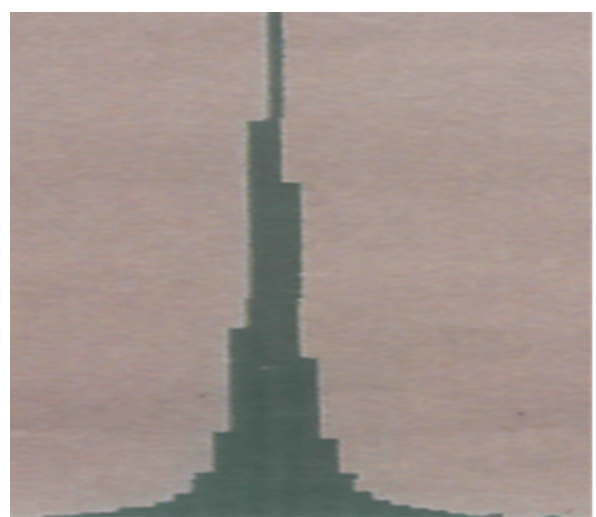

Figure 2. Point-spread function experimentally obtained

\section{MATHEMATICAL PROBLEM IN PET}

In PET, let the back-projected image $\mathbf{D}=\left\{d_{i}\right\}(i=1, \ldots, M)$ be relevant to a given plan which consists of $M$ pixels, and the density of these pixels is a set of positive numbers $\left\{f_{j}\right\}(j=1, \ldots M)$. If $\left\{R_{i j}\right\}$ represents the physical connection between the density in a plane and the mock data it would produce, the image formation equation can be implemented in the form:

$$
d_{i}=\sum_{j=1}^{M} R_{i j} f_{j},(i=1,2,3, \ldots, N) .
$$

The point-spread function $R_{i j} \quad$ obtained experimentally and shown in Figure 2 is proportional to the probability that a photon pair emitted from the $j$ th pixel is detected by the $i$ th tube. It is assumed to be modelled correctly, e.g., sampling effects and scattering contributions are properly incorporated. Given the back-projected data $\mathbf{D}$ and the knowledge of $\mathbf{R}$, the mathematical problem of PET imaging is to estimate $\mathbf{f}$. Mathematically, such an inverse problem is an ill-posed one, making the solution non-trivial and ambiguous. This is because very small variations in the experimental data $\mathbf{D}$ lead to very large variations in the solution $\mathbf{f}$. Therefore, it generally requires some kind of regularization in order to generate physically plausible reconstructions. This can be done in a variety of ways $[18,19]$, but a commonly used idea to realize regularization techniques with statistical motivation is the Bayesian approach that is based on the probability theory, which makes it possible to rank a continuum of possibilities on the basis of their relative likelihood $p(\mathbf{D} \mid \mathbf{f})$ or preference and to conduct inference $p(\mathbf{f})$ in a logically consistent way. From Bayes' formula, we 
obtain the posterior probability density function (PDF) $p(\mathbf{f} \mid \mathbf{D})$ in the following form:

$$
p(\mathbf{f} \mid \mathbf{D})=\frac{p(\mathbf{D} \mid \mathbf{f}) p(\mathbf{f})}{p(\mathbf{D})},
$$

where $p$ (D) may be considered necessary only for normalization purposes. Thus, additional prior information on $\mathbf{f}$ via the a-priori PDF $p(\mathbf{f})$ is put into the reconstruction process. According to Skilling [21], the prior PDF $p(\mathbf{f})$ for a positive additive image $\mathbf{f}$ is proportional to $\exp (\alpha S(\mathbf{f}, \mathbf{m}))$, where the entropy $S(\mathbf{f}, \mathbf{m})$ of the required image $\mathbf{f}$, relative to an initial model $\mathbf{m}$ is defined by

$$
S(\mathbf{f}, \mathbf{m})=\sum_{j=1}^{N} f_{i}-m_{j}-f_{j} \log \left(\frac{f_{j}}{m_{j}}\right) .
$$

Here $\alpha$ is a regularization parameter, which is initially undetermined but its value is estimated along the iteration process. For a Poisson-distributed event counting experiment, the conditional probability of the whole data set given is

$$
p(\mathbf{D} \mid \mathbf{f})=\frac{1}{\prod_{i=1}^{N} \sqrt{2 \pi} \sigma_{i}} \exp \left(-\frac{\chi^{2}(\mathbf{f})}{2}\right),
$$

where $\chi^{2}(\mathbf{f})$ is usually used as a measure of the misfit:

$$
\chi^{2}(\mathbf{f})=\sum_{i=1}^{M} \frac{1}{\sigma_{i}^{2}}\left(d_{i}-\sum_{j=1}^{N} R_{i j} f_{j}\right)^{2} .
$$

Here $\sigma_{i}$ is the standard deviation of the noise in the $i$ th pixel. Then, the posterior PDF in Equation (2) turns into the following form:

$$
p(\mathbf{f} \mid \mathbf{D}) \approx \exp \left(\alpha S(\mathbf{f}, \mathbf{m})-\frac{1}{2} \chi^{2}(\mathbf{f})\right)
$$

The computationally interesting Bayesian approach called the maximum a-posteriori probability (MAP) estimation consists of computing an estimate $\mathbf{f}$ of the unknown object by maximizing the a-posteriori PDF $p(\mathbf{f} \mid \mathbf{D})$ given in Equation (6). Various numerical algorithms for solving this non-linear optimization problem have been suggested by various researchers $[17,18,19]$. However, we shall only consider here a complicate but highly successful scheme developed by Skilling and collaborators [20], wherein a maximum is repeatedly sought not along a single search direction but in a small dimensional subspace, spanned by vectors that are calculated at each landing point. The subspace's basis vectors are chosen in such a way as to avoid directions leading to negative values. One of the most successful choices is the three-dimensional subspace spanned by the following vectors with components given by

$$
\begin{aligned}
& \mathbf{e}_{1}=\mathbf{f}(\nabla S) \\
& \mathbf{e}_{2}=\mathbf{f}\left(\nabla \chi^{2}\right) \\
& \mathbf{e}_{3}=|\nabla S|^{-1} \mathbf{f}\left(\nabla^{2} \chi^{2}\right) \mathbf{f}(\nabla S)-\left|\nabla \chi^{2}\right|^{-1} \mathbf{f}\left(\nabla^{2} \chi^{2}\right) \mathbf{f}\left(\nabla \chi^{2}\right)
\end{aligned}
$$

The entropy metric (- $\tilde{\mathrm{N}} \tilde{\mathrm{N}} S$ ) is used here to define the following lengths:

$$
|\nabla S|=\left(\sum f_{i}\left(\frac{\partial S}{\partial f_{i}}\right)^{2}\right)^{\frac{1}{2}},\left|\chi^{2}\right|=\left(\sum f_{i}\left(\frac{\partial \chi^{2}}{\partial f_{i}}\right)^{2}\right)^{\frac{1}{2}}
$$

With these three search directions, quadratic models for $S(\mathbf{f}, \mathbf{m})$ and $\chi^{2}(\mathbf{f})$ are constructed in the subspace given in Equation (7) and then the algorithm proceeds by maximizing the entropy prior $S(\mathbf{f}, \mathbf{m})$ subject to the constraint $\chi^{2}(\mathbf{f}) \leq N$ by implicitly adjusting $\alpha$. More precisely, in each iteration, the entropy $S(\mathbf{f}, \mathbf{m})$ and the misfit $\chi^{2}(\mathbf{f})$ are projected onto a three-dimensional subspace where $S(\mathbf{f}, \mathbf{m})$ attains its maximum while $\chi^{2}(\mathbf{f}) \leq N$ is satisfied so that the iteration is repeated until the following criterion is satisfied:

$$
T E S T=\frac{1}{2}\left|\frac{\nabla S}{|\nabla S|}-\frac{\nabla \chi^{2}}{\left|\nabla \chi^{2}\right|}\right|^{2}<\varepsilon,
$$

where $\varepsilon$ is a preassigned value specified by a user.

\section{COMPUTER SIMULATIONS}

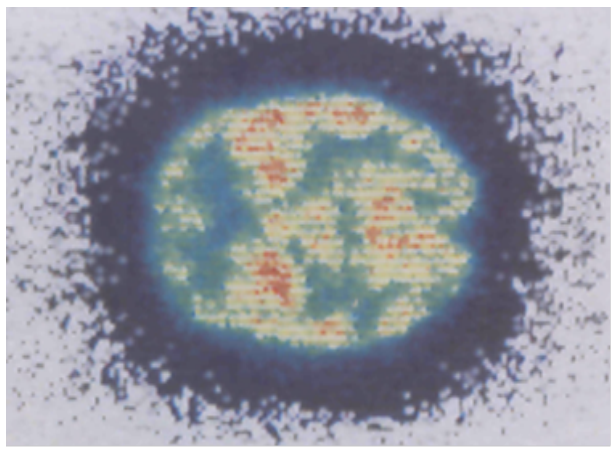

(a)

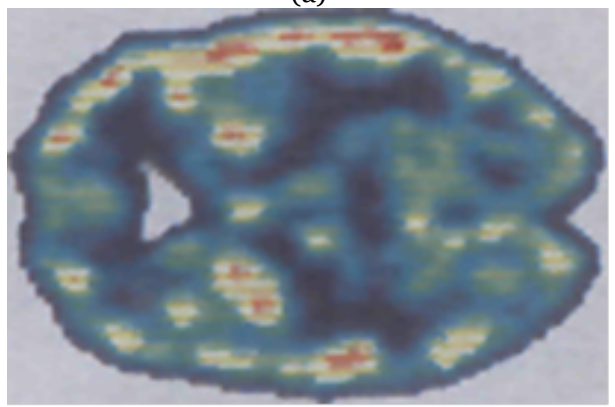

(b)

Figure 3. (a) The back-projected data; (b) the reconstructed image formed by the CMEP 
The concentration of a chemical compound, labelled gallium isotope $\left({ }^{68} \mathrm{Ga}\right)$ with the half-life of 68 minutes, was measured in the brain by using the PET camera and was provided by the positron emission group at the School of Physics and Space Research at the University of Birmingham. What the PET camera records is a pair of coordinate values- one from each detector represents each annihilation event. By using that recorded pair of coordinate values, the backprojected images of the brain shown in Figures 3(a) and $4($ a) were constructed.

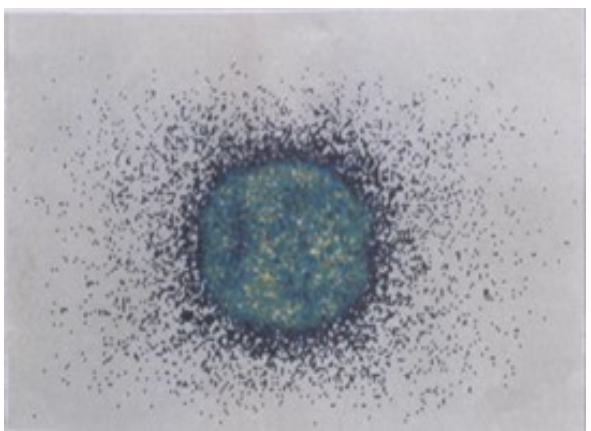

(a)

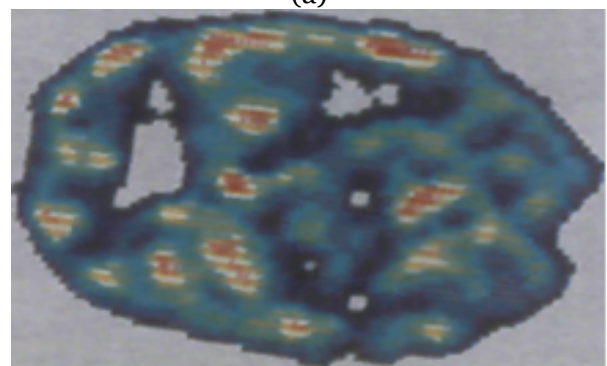

(b)

Figure 4. (a) the back-projected data; (b) the image reconstructed by the CMEP

Imaging or data analysis problems of PET camera are only considered here, but it is known that such studies provide quantitative functional information, which helps to quantify normal human brain physiology and highlights the changes in metabolism and function caused by cerebral disease.

The images shown in Figures 3(a) and 4(a) are the sectional images of the brain that are the colour-coded to show differences in the level of activity from point to point. The number of random coincidence events collected by the PET camera distinguishes these two sets of data, taken from the central plane. This backprojected data in Figure 4(a) was therefore obtained by discarding $10 \%$ of information from the back-projected data given in Figure 3(a). It is expected that the more counts collected, the better the image quality that results. The CMEP requires a specification of a model image and the variance of the noise in the data. The model image $\mathbf{m}$ represents our prior state of knowledge or ignorance about the original image. It is usually taken to be uniform but the use of a nonuniform model image allows us to introduce partial correlations across the image we want to infer. Therefore, at the $k$ th iteration, we estimated the model $\mathbf{m}^{k}$ in the form:

$$
\mathbf{m}^{k}=\mathbf{B} \mathbf{f}^{k},
$$

where $\mathbf{B}$ is a blurring matrix, which averages the densities over neighboring pixels $(3 \times 3)$. The uncertainty in the data associated with each pixel varies from pixel to pixel according to the number of photons counted. Therefore, the variance of the noise $\sigma_{i}^{2}$ was estimated in the form:

$$
\sigma_{i}^{2}=\left\{\begin{array}{cl}
d_{i} & \text { if } d_{i} \neq 0 \\
1 & \text { if } d_{i}=0
\end{array}, \quad i=1, \ldots, M .\right.
$$

A fictional photon is added here to avoid the discontinuity in the region where $d_{i}=0$. The CMEP was run on an IBM3090 computer and computer simulations are tabulated in Table 1 . The colour-coded images of the reconstructed densities are shown in Figures $3(\mathbf{b})$ and $4(\mathbf{b})$. The red areas in the pictures indicate high levels of activity; dark blue or white areas indicate little or no activity in those brain structures. It is also observed that they have almost similar brain structures even though the data shown in Figure 4(a) contain less information than the one in Figure 3(a). It is seen that the quality of reconstruction is better when the statistics is good in data. As expected, CMEP requires a large consumption of CPU time on the computer because it is non-linear and iterative.

Table 1. Computer Simulations of CMEM

\begin{tabular}{ccccc}
\hline Figure & $k$ & $S$ & TEST & CPU \\
\hline 1 & 50 & -31670.0 & 0.0002 & $7(\mathrm{~h})$ \\
\hline 2 & 30 & -26160.6 & 0.0009 & $5(\mathrm{~h})$ \\
\hline
\end{tabular}

\section{CONCLUSIONS}

In this work, CMEM is used to reconstruct PET images with Poisson noise model including partial correlations. It is mainly based on a subspace of several search directions instead of a line search. The noise level that constrains over the likelihood function is controlled so that the positivity during each subspace search is directly enforced to the reconstructions. These features make the algorithm attractive for solving high-dimensional nonlinear problems. Overall, PET reconstructions indicate that the application of the maximum entropy method leads to considerably improved reconstruction, especially in the case where the data are very noisy and the sampling incomplete. Along with improving the reconstruction methods for solving the basic PET reconstruction problem, there are many novel challenges facing this field and new advances in PET instrumentation require hybrid reconstruction algorithms to reach their potential improvements in signal-to-noise ratio. Clinical importance of PET imaging will continue to increase.

Acknowledgement: I would like to express our thanks to Dr. Hawkesworth and Dr. D. Paker for 
providing the back-projected PET data and to Dr. J. Skilling for sending us the Cambridge MaxEnt Package and for his comments about the results. This work is a part of project whose name is "Positron lifetimes spectrum analysis with Bayesian statistical inference" with a number FEN-A-06051O-0135 supported by Marmara University, Istanbul, Turkey.

\section{REFERENCES}

1. A. M. Cormack, "Representation of a function by its line integral, with some radiological applications II," J. App. Physics, vol. 35, no. 10, pp. 2908 - 2913, Oct. 1964.

DOI: $10.1063 / 1.1713127$

2. G. N. Hounsfield, "Computerized transverse axial scanning (tomography). I: Description of System," Brittan Journal of Radiology, vol. 46, no. 552, pp. 1016 - 1022, Dec. 1973.

DOI: $10.1259 / 0007-1285-46-552-1016$ PMid: 4757352

3. J. Radon, "Über die bestimmung von funktionen durch ihre integralwerte längs gewisser mä nnigfaltigkeiten," Akad. Wiss., vol. 69, pp. 262 - 277, Apr. 1917. (J. Radon. "On the determination of functions by their integrals along certain manifolds," Acad. Sci., vol. 69, pp. 262 - 277, Apr. 1917.)

Retrieved from:

http://people.csail.mit.edu/bkph/courses/papers/E xact Conebeam/Radon Deutsch 1917.pdf

Retrieved on: Jan. 20, 2017

4. N. M. Ter-Pogossian, M. E. Raichle, B. E. Sobel, "Positron-emission tomography," Scientific America vol. 243, no. 4, pp. 170-181, Oct. 1980.

DOI: $10.1038 /$ scientificamerican1080-170

PMid: 6821228

5. J. Verhaeghe, A.J. Reader, "Simultaneous water activation and glucose metabolic rate imaging with PET," Phys. Med. Biol., vol. 58, no. 3, pp. 393 - 411, Feb. 2013.

DOI: 10.1088/0031-9155/58/3/393

PMid: 23296197

6. R. J. Ott, M. A. Flower, J. W. Babich, P. K. Marsden, "The physics of radioisotope imaging," in The Physics of Medical Imaging, S. Webb, Ed., 1st ed., Bristol, UK: Adam Hilger, 1988, ch. 6, pp. $142-318$. DOI: $10.1201 / 9781439822081$

7. G. L. Zeng, Medical Image Reconstruction: A Conceptual Tutorial, Berlin, Germany: SpringerVerlag, 2010.

DOI: $10.1007 / 978-3-642-05368-9$

8. I. C. Smith, A. E. Welch, F. Chilcott, S. D. Heys, P. Sharp, O. Eremin, "Gamma Emission Imaging in The Management of Breast Disorders," European $J$. of Surgical Oncology, vol. 24, no. 4, pp. $320-329$, Aug. 1998. DOI: $10.1016 /$ So748-7983(98)80016-4

9. J. S. Lee, "Technical Advances in Current PET and Hybrid Imaging Systems," The Open Nuclear Medicine Journal, vol. 2, pp. 192 - 208, Dec. 2010. DOI: $10.2174 / 1876388 X 01002010192$

10. M. J. Ehrhardt, K. Thielemans et al., "Joint reconstruction of PET-MRI by exploiting structural similarity," Inverse Problems, vol. 31, no. 1, pp. 1 23, Jan. 2015.

DOI: $10.1088 / 0266-5611 / 31 / 1 / 015001$
11. A. DelGuerra, N. Belcari, M. Bisogni, "Positron emission tomography: its 65 years," Riv. Nuovo Cimento, vol. 39, no. 4, pp. 155 - 223, Apr. 2016. DOI: $10.1393 / \mathrm{ncr} / \mathrm{i} 2016-10122-6$

12. H. Zaidi, M. Becker, "The Promise of hybrid PET/MRI: technical advances and clinical applications," IEEE Signal Process Mag., vol. 33, no. 3, pp. 67-85, Apr. 2016. DOI: 10.1109/MSP.2015.2482225

13. V. Bettinardi, L. Presotto, E. Rapisarda, "Physical performance of the new hybrid PETCT discovery690.," Med. Phys., vol. 38, no. 10, pp. 5394 - 5411, Oct. 2011.

DOI: $10.1118 / 1.3635220$

PMid: 21992359

14. G. Wang and J. Qi, "An optimization transfer algorithm for nonlinear parametric image reconstruction from dynamic PET data," IEEE Trans. Med. Imaging, vol. 31, no. 10, pp. 1977 1988, Oct. 2012.

DOI: 10.1109/TMI.2012.2212203

PMid: 22893380 PMCid: PMC4086832

15. R. M. Leahy and J. Qi, "Statistical Approaches in Quantitative Positron Emission Tomography," Statistics and Computing, vol. 10, no. 2, pp. 147 165, Apr. 2000 DOI: 10.1023/A:1008946426658

16. R. M. Lewitt, S. Matej, "Overview of methods for image reconstruction from projections in emission computed tomography," Proc. IEEE Inst. Electr. Electron Eng., vol. 91, no. 10, pp. 1588 - 1611, Oct. 2003. DOI: $10.1109 / J P R O C .2003 .817882$

17. K. M. Hanson, "Introduction to Bayesian image analysis," in Medical Imaging: Image Processing, M. H. Loew, Ed., Newport Beach (CA), USA: SPIE 1898, 1993, pp. $716-731$. Retrieved from: http://kmhlanl.hansonhub.com/publications/medim93.pdf Retrieved on: Jan. 20, 2017

18. J. Qi, R. M. Leahy, "Iterative reconstruction techniques in emission computed tomography," Phys. Med. Biol. vol. 51, no. 15, pp. $541-578$, Aug. 2006.

DOI: $10.1088 / 0031-9155 / 51 / 15 /$ Ro1 PMid: 16861768

19. A. R. Davies, R. S. Abderssen, "Optimization in the regularization of Ill-Posed Problems," J. Austr. Math. Soc. Series B, vol. 28, no. 1, pp. 114 - 133, Jul. 1986. DOI: $10.1017 /$ So334270000005221

20. J. Skilling, R. K. Bryan, "Maximum entropy image reconstruction: General algorithm," Monthly National Radio Astronomy Society, vol. 211, no. 1, pp. $111-124$, Nov. 1984. DOI: 10.1093/mnras/211.1.111

21. J. Skilling, "Quantified maximum entropy," in Maximum Entropy and Bayesian Methods, F. Fougère, Ed., Dordrecht, The Netherlands: Kluwer Academic Publishers, 1990, ch. 12, pp. 341 - 351. DOI: 10.1007/978-94-009-0683-9_21 